\title{
POUR COMPRENDRE LA VIOLENCE: L'HYPOTHÈSE DU SUJET
}

\section{Michel Wieviorka*}

\begin{abstract}
Resumo: O objetivo do texto é esboçar uma teoria da violência centrada na noção de sujeito. A teorização proposta não visa a descartar outras perspectivas de abordagem; não pretende opor o ponto de vista do sujeito a outros pontos de vista, tais como aqueles centrados, por exemplo, na racionalidade do ator ou em suas frustrações, mas, ao contrário, situar esses outros pontos de vista no contexto de um enfoque mais amplo, no qual sejam paralelos ou, até mesmo, subordinados à noção de sujeito. Privilegiar o ponto de vista do sujeito significa, fundamentalmente, utilizar dois caminhos que frequientemente se cruzam mas que, para os objetivos da análise, necessitam ser dissociados: o primeiro concentra-se nos protagonistas da violência; o segundo, em suas vítimas. O ponto de partida é uma definição da noção de sujeito, uma vez que, freqüentemente, a violência é a marca de um sujeito contrariado, proibido, impossível ou infeliz, a marca de uma pessoa que foi, ela mesma, vítima de uma violência.
\end{abstract}

Palavras-chave: violência urbana, sociologia do sujeito, conflitualidade, construção de sentido, teoria da violência.

Dès qu'apparaît le mot de «violence», d'innombrables problèmes surgissent, à commencer par celui de savoir de quoi nous parlons. Car le terme de violence amalgame un immense ensemble de notions, elles-mêmes plus ou moins confuses ou embrouillées. Violence physique, ou aussi symbolique? Réelle, ou perçue? Individuelle, ou collective? Violence d'Etat, éventuellement légitime, ou violence de la rue, à la légitimité contestable? Etc. Cette liste de couples d'opposition, qui ne sont pas nécessairement contradictoires, pourrait être allongée considérablement, et on pourrait en ajouter

\footnotetext{
* Sociólogo; professor da École des Hautes Études en Sciences Sociales - EHESS/Paris; diretor do Centre d'Analyse et d'Intervention Sociologiques (CADIS).

Artigo recebido em 16 abr. 2003; aprovado em 21 mai. 2004.
} 
d'autres, fondées par exemple sur des catégories empiriques, et qui peuvent elles aussi être déclinées à l'infini: en fonction des auteurs violence ouvrière, ethnique, islamique, paysanne, des jeunes, etc. -, en fonction des cibles ou des victimes - les jeunes, les femmes, les minorités ethniques, etc. -, en fonction des domaines de la vie collective - violence urbaine, violence rurale, violence sociale, violence politique, etc.

Le mot de violence ne fusionne pas seulement des phénomènes distincts, ou qu'il faudrait distinguer analytiquement, il autorise aussi la mobilisation, plus ou moins confuse là encore, de toutes les théories générales disponibles dans les sciences sociales et dans la philosophie politique, sans parler de théorisations spécifiques, apportant un éclairage ad hoc plus ou moins lumineux. On ne s'étonnera pas, dès lors, de trouver, dans la littérature des sciences sociales, des ouvrages qui se proposent de passer en revue les diverses théories disponibles, ${ }^{1}$ ou d'autres qui ont pour principale caractéristique de tenter avec plus ou moins de bonheur d'en articuler ou d'en intégrer plusieurs, par exemple pour venir au secours d'un mode d'approche principal manifestement défaillant, tant les faits requièrent d'autres paradigmes. $^{2}$

Ma réflexion, ici, circulera au sein d'un ensemble non ou peu délimité d'expériences historiques concrètes de violence, elle passera d'un objet empirique à un autre, sans avoir le souci d'éclairer plus systématiquement telle ou telle famille de violence, sans véritablement déconstruire ou dissocier ce que le terme même agrège d'une manière qui constitue souvent un obstacle à la compréhension. Mais elle le fera en refusant l'éclectisme théorique, et donc la juxtaposition de modes d'approche sans unité, en privilégiant, au contraire, une entrée bien délimitée, un point de vue spécifique. Le pari que j'entends prendre ici est en effet celui de développer un raisonnement à la fois peu courant dans les sciences sociales, du moins s'il s'agit d'aborder la violence, et centré sur une idée-force à partir de laquelle se construit la mise en perspective. Disons, en un mot, que l'enjeu de ces réflexions est d'esquisser une théorie de la violence reposant sur la notion de sujet. Pourtant, la théorisation que je vais ébaucher n'écarte pas 
systématiquement d'autres modes d'approche; en particulier, elle entend non pas opposer le point de vue du sujet à d'autres, centrés par exemple sur la rationalité de l'acteur, ou sur ses frustrations, et qui peuvent s'avérer d'une certaine utilité, mais situer ces autres points de vue dans le cadre d'un raisonnement plus large où ils sont en quelque sorte latéraux, voire subordonnés à la notion de sujet.

Dire que j'entends privilégier le point de vue du sujet, c'est pour l'essentiel envisager d'emprunter deux chemins, appelés à se croiser fréquemment, mais qu'il faut dissocier pour l'analyse. Le premier s'intéresse aux protagonistes de la violence, ceux qui y passent, la pratiquent, s'y installent, en sortent aussi, éventuellement. Le second, que je ne pourrai ici qu'envisager plus rapidement, renvoie à ses victimes, ceux qu'elle vise, atteint et affecte, directement ou indirectement.

Mais peut-être faut-il au préalable apporter une définition, même rapide, de la notion de sujet telle que je l'utiliserai ici. Le sujet, c'est la capacité de se construire soi-même, de procéder à des choix, de produire sa propre existence. C'est, pour reprendre le vocabulaire de Hans Joas (1999 [1992], p. 150), "le caractère créateur de l'agir humain". C'est la capacité de s'engager, et donc tout aussi bien, de se dégager. Et il n'est de sujet que dans la reconnaissance du sujet chez l'Autre, dans l'acceptation de l'altérité. Le sujet n'est pas pour autant un électron libre, dont la trajectoire personnelle échapperait à toute contrainte, à toute norme, à toute relation avec d'autres que celles qu'il choisirait. Il n'existe que dans la capacité à vivre des relations. Je pense même qu'il y a d'autant plus d'espace ouvert au sujet qu'il y a, précisément, dans une société, des possibilités de reconnaissance à l'œuvre aussi bien dans les relations interpersonnelles (en particulier dans la famille) que dans la communication interculturelle, et dans le cadre de rapports sociaux, y compris et surtout, j'y reviendrai, sur un mode conflictuel. Le sujet n'est qu'une dimension de la personne, que je distinguerai de l'individu, défini à mes yeux non pas par la production de soi-même, mais par la participation à la modernité et notamment par la consommation et l'accès à l'argent. Enfin, le sujet est pour moi une 
catégorie abstraite, qui trouve sa réalisation concrète la plus importante dans l'action. Mais le sujet ne devient pas toujours ou facilement ou pleinement acteur, et c'est même souvent dans l'espace qui sépare le sujet de l'acteur que s'ébauche la violence.

Formulons en termes trop simples, mais qui peuvent constituer un solide point de départ, notre hypothèse principale: la violence est souvent, au moins partiellement, au moins à l'origine, la marque d'un sujet contrarié, interdit, impossible ou malheureux. La marque, éventuellement, d'une personne ayant elle-même subi une violence soit physique, comme ces traumatisés de la guerre qui développent ensuite des troubles de personnalité pouvant inclure des épisodes de violence, soit morale ou symbolique, comme c'est souvent le cas pour les jeunes délinquants. ${ }^{3}$

\section{L'expérience des violences urbaines}

Une telle formulation nous autorise à intégrer dans notre raisonnement le thème de la frustration, si courant dans l'analyse de la violence : celle-ci, en effet, peut procéder d'une frustration, au sens où une personne, ou un groupe se voient privés ou interdits d'accès à des biens matériels ou à une reconnaissance symbolique légitimes. La frustration, par exemple, peut être celle du jeune qui devient délinquant pour accéder à la consommation, et qui, comme l'a très bien dit Robert Merton, emploie des moyens illégitimes pour parvenir à des fins légitimes, et se conformer aux valeurs d'une société qui attribue à l' argent et aux biens matériels une importance considérable. La frustration, ici, semble renvoyer davantage à l'individu soucieux de consommer, qu'au sujet s'efforçant de se construire. Mais déjà là, le thème du sujet peut apporter un éclairage utile, en suggérant que derrière l'action pour accéder à des biens ou à de l'argent, il y a un désir de paraître, de s'affirmer, de gagner l'estime de soi et peut-être celle des autres, ou de certains autres. Et surtout, dès qu'on associe la frustration au thème de la reconnaissance, on conçoit vite l'importance 
de la place du sujet. C'est ce que nous allons voir en considérant un phénomène relativement bien délimité, les émeutes urbaines, telles qu'elles se sont développées en France à partir du début des années quatre-vingt, ainsi d'ailleurs qu'en Grande-Bretagne.

\section{La demande de reconnaissance}

La frustration du jeune qui passe à l'émeute, ou qui se comporte comme un enragé, conduites qui sont au cœur des représentations de la violence dite urbaine, en effet, est constamment informée par un vif sentiment d'injustice, de non-reconnaissance, par la conviction de vivre dans une société qui, comme le disait un des principaux protagonistes de la vague terroriste de 1995 en France, Khaled Kelkal, trois ans avant sa dérive puis sa mort, ne «laisse pas sa place». La violence urbaine explose lorsque cette négation de la personne comme sujet, vécue comme particulièrement douloureuse par des jeunes sans grand avenir et soumis à la discrimination sociale et au racisme, est soulignée par le comportement de la police ou les décisions de la justice, par une « bavure » policière qui aboutit à la mort d'un jeune du quartier, par l'acquittement de policiers coupables de graves brutalités - rappelons que la grande émeute de Los Angeles en avril 1992 a été déclenchée par l'acquittement des policiers blancs accusés, bande vidéo à l'appui, d'avoir battu sauvagement Rodney King, un noir qui ne les avait en aucune façon menacés. L'expérience des émeutes urbaines contemporaines nous invite à ne pas nous satisfaire trop facilement du vocabulaire de la frustration, ou en tout cas à dire la nature de la frustration envisagée: ici est en jeu un déni de subjectivité, à la limite une négation par le racisme de l'humanité de ceux qui en définitive se révoltent, une profonde injustice qui touche à l'intégrité morale de la personne.

\section{La critique des institutions}

Mais une autre dimension de ces violences, particulièrement marquée en France, nous conduit à compléter l'analyse: en France, plus qu' ailleurs, au point qu' on peut se demander s'il n'y a pas là une 
expression originale de l'"exception française", ${ }^{4}$ la violence urbaine s'en prend constamment aux institutions et à ceux qui les incarnent, elle affecte l'école publique, les transports publics, les équipements collectifs, y compris s'ils sont mis à la disposition des jeunes des quartiers, sans parler de la police et de la justice, elle vise même les pompiers ou les travailleurs sociaux de plus en plus souvent. Elle traduit la colère, le ressentiment, et le sentiment d'être victimes de mensonges et d'abandon de ceux qui constatent que les belles promesses de la République - liberté, égalité, fraternité - ne sont pas tenues, du moins en ce qui les concerne. Dans un pays où de nombreux acteurs politiques et certains intellectuels répètent de façon presque incantatoire le credo républicain, alors qu'il devient inopérant pour une partie de la population, la violence exprime une subjectivité sans débouchés, l'incapacité d'avoir des projets, d'agir de manière créatrice, et de produire son existence, elle vient souligner le fossé qui sépare les institutions de ceux à qui elles devraient apporter les chances et les moyens de se construire.

\section{La crise des institutions}

Mais avançons d'un pas encore, en continuant à tirer les leçons d'une expérience de recherche qui a porté précisément sur les violences urbaines en France. Si dans ce pays, l'émeute ou la rage, ou d'autres conduites encore s'en prennent aux institutions, ce n'est pas seulement parce que la société dans son ensemble, ou ses élites politiques et intellectuelles, tiennent des discours et font des promesses républicaines démesurées par rapport à ce qui est réellement offert. C'est aussi parce que ces institutions sont en crise et génèrent des conduites, en leur sein même, qui sont la marque pour leur personnel de la négation du sujet. Les institutions de la République, en effet, ont longtemps incarné l'intégration réussie de trois enjeux distincts : elles sont supposées tout à la fois, premièrement, répondre à l'intérêt supérieur de la collectivité, que celui-ci soit défini en termes de service public, de solidarité, d'égalité, ou bien encore en termes plus culturels de nation, deuxièmement, maintenir leur propre intégrité et leur 
capacité de fonctionnement, et troisièmement, apporter à leurs personnels des garanties et un statut, matériel et symbolique, bien plus gratifiant que s'ils travaillaient pour le secteur privé. Or tout ceci n'a cessé de se désintégrer depuis la fin des années soixante. Et dans cette déstructuration, les personnels sont mal à l'aise, inquiets, tentés par le retrait, le corporatisme exacerbé, mais aussi par l'égoïsme, voire le racisme. Ils leur devient difficile de s'identifier à des intérêts qui les transcendent, de se vivre aussi bien que par le passé comme des sujets en prise directe avec l'universel. Et leurs comportements, s'il s'agit de ceux qui sont en contact avec les quartiers populaires ou leurs habitants, tout comme leurs décisions, à tous les niveaux, peuvent prendre l'allure de la négation de ce qu'il y a ou pourrait y avoir de subjectivité en face, chez ceux dont ils ont la charge, directement ou indirectement. La spirale de la violence dite urbaine, lorsqu'elle est en réalité scolaire, ou qu'elle vise les transports publics, doit ainsi beaucoup à des transformations dans lesquelles des sujets malheureux, des personnels inquiets, ou raidis sur leurs intérêts limités, des directions perdant le sens des demandes et des attentes de la population contribuent, depuis leurs institutions, à produire au dehors de la subjectivité elle aussi malheureuse ou impossible. Rien n'est plus parlant ici que le non-dialogue que l'on observe couramment sur le terrain entre policiers et jeunes des banlieues dites difficiles ou d'exil: les uns et les autres, en effet, se plaignent de la même chose, ils disent ne pas être entendus ou reconnus, être victimes d'un racisme - antiflics et anti-français pour les uns, anti-arabes ou anti-immigrés, mais aussi anti-jeunes pour les autres. Et dans cette non-reconnaissance mutuelle, la violence trouve vite son chemin.

Cette remarque empirique a des implications théoriques qu'il ne faudrait pas sous-estimer: elle signifie, en effet, qu'il est possible, à propos ici de la violence, d'inscrire une sociologie des organisations, et de leur crise, dans le cadre d'une sociologie du sujet. Et ces implications théoriques peuvent avoir des prolongements politiques, car si on accepte, en matière de réformes des institutions, de prendre le point de vue du sujet, il est possible d'élaborer des propositions bien différentes de celles qu'offrent les perspectives plus classiques. 


\section{Violence et conflit}

Le sujet, nous l'avons dit, n'est pas un électron libre. Et ce n'est pas un paradoxe que d'affirmer qu'il a d'autant plus de chances de se construire et de s'exprimer qu'il est partie prenante de relations, y compris et surtout lorsqu'elles sont de l'ordre du conflit. Mais avant de développer ce paradoxe, peut-être faut-il d'abord donner une signification précise à ce mot de conflit, qui renvoie pour moi à un rapport, structuré de façon plus ou moins stable et durable. Les acteurs qui s'opposent dans un conflit sont des adversaires, et non pas des ennemis, et même si tout n'est pas négociable dans un conflit, celuici est le contraire de la violence, qui ferme l'espace de la discussion et du débat au profit de la rupture ou du seul rapport de force.

\section{L'expérience du mouvement ouvrier}

Lorsqu'une société est structurée par un conflit central, comme ce fut le cas tout au long de l'ère industrielle classique, la brutalité plus ou moins marquée de la domination et de l'exploitation alimente non pas tant des conduites de violence qu' une contestation qui cherche à s'organiser sur le long terme, des luttes, des engagements qui peuvent aboutir à des revendications négociées, des pressions politiques, des mouvements sociaux portés par un projet reposant sur la subjectivité des acteurs. Le mouvement ouvrier procède de la privation ou de la dépossession dont souffrent les ouvriers à qui il est interdit ou difficile d'avoir la maîtrise de leurs œuvres, de contrôler la production ; mais il est aussi défini par un projet et l'affirmation d'une subjectivité, certes malheureuse. Les ouvriers, surtout s'ils sont qualifiés, ont une certaine fierté, la conscience d'avoir un rôle social, même plus ou moins aliéné, de mériter le respect, de ne pas faillir à l'estime de soi. Et plus ils sont capables de mener une action à la fois défensive et contre-offensive, moins ils se tournent vers des conduites de violence, de destruction ou d'autodestruction.

Tout change avec la sortie de la société industrielle. D'une part, ceux qui subissent le choc de la désindustrialisation, de la perte 
d'emploi, du chômage, de l'exclusion ou de la précarité perdent aussi les repères qui autorisaient une image positive d'eux-mêmes, même s'ils étaient exploités et dominés. Ils sont plus susceptibles, eux ou leurs enfants, de passer à la violence. On sait bien, depuis les années quatre-vingt, que les conduites juvéniles de violence, dans les quartiers en crise, doivent beaucoup à ce déclin du conflit caractéristique de l'ère industrielle, notamment dans les anciennes banlieues rouges. Se met alors en place, plus facilement, une culture de winner-loser, où le mépris et sa hantise viennent occuper une place considérable : pour ceux qui sont "jetables", comme on dit souvent en Amérique latine, ou rejetés, il se développe un vif sentiment de déchoir et l'absence ou la perte d'estime de soi peut envahir la conscience. N'eston pas inutile socialement, mis au rebut ou presque ? Et dans ce contexte, la violence trouve sa voie bien plus facilement que dans une culture ouvrière où le sentiment d'être dominé et exploité n'interdit pas, au contraire, la conscience d'une utilité sociale.

D'autre part, lorsqu'il existe de fortes communautés ouvrières, une vie sociale dense, structurée par le conflit central propre aux sociétés industrielles, lorsque, à partir du mouvement ouvrier, se mettent en place des formes de vie politique, un tissu associatif et des débats d'idées, la violence, du moins dans ses formes les moins graves, n'est pas perçue comme aussi dangereuse ou insupportable que lorsque la vie sociale est décomposée, sans repères, et que la moindre agressivité - les « incivilités » dont se repaît depuis quelques années la littérature spécialisée dans l'insécurité - déclenche un vif sentiment de peur et de menace.

\section{En amont et en aval du conflit}

Sans aller jusqu'à en faire une théorie générale ou un principe unique d'analyse, il est possible d'étendre les remarques concrètes qui précèdent pour proposer un raisonnement associant de manière plus large violence et conflit, l'un étant en quelque sorte le contraire de l'autre. La conséquence de cette idée de base est que l'espace de la violence, en bonne logique, doit être d'autant plus vaste que celui 
du conflit lui-même rétrécit. On peut ainsi considérer que certaines manifestations de violence, ou certaines significations à l'œuvre dans telle ou telle expérience de violence, expriment la faiblesse d'un conflit, ou bien parce que celui-ci, naissant, n'est pas encore pleinement constitué et perçu comme tel par ses protagonistes et dans la société où il s'ébauche ; ou bien parce que, à l'opposé, il est dans une phase de crise, de déstructuration ou de déclin historique; ou bien encore, parce que les deux logiques de faiblesse, en amont et en aval du conflit, se conjuguent.

On peut illustrer cette idée par le rappel de quelques expériences terroristes $^{5}$. Le terrorisme anarchiste en France, entre 1892 et 1894 , l' «ère des attentats», comme a dit Jean Maîtron, exprime en creux la faiblesse d'un conflit émergent, il annonce la naissance d'un mouvement social, et, plus précisément, la formation d'un acteur syndical capable d'une réelle mobilisation. Il cesse exactement en même temps que s'affirme, dans les Bourses du Travail et dans les syndicats, la première grande expression organisée du mouvement ouvrier en France, le syndicalisme d'action directe, parfois appelé aussi syndicalisme révolutionnaire ou anarcho-syndicalisme. Symétriquement, le terrorisme d'extrême gauche, dans de nombreux pays occidentaux, vient exprimer dans les années soixante-dix et quatre-vingt, sur le mode que j'ai appelé de l'inversion, la fin du mouvement ouvrier et des idéologies qui en faisaient le sel de la terre, ses protagonistes essayant de maintenir au plus haut niveau, et de plus en plus artificiellement, le sens d'une action en déclin. Et dans certains cas, le même terrorisme est chargé non seulement de significations qui renvoient à la fin du conflit classique de l'ère industrielle, mais aussi, c'est très net en Italie, à la naissance timide de nouvelles sensibilités culturelles, à l'ébauche de nouveaux mouvements sociaux, à de nouvelles subjectivités trop faibles pour s'affirmer de manière autonome, et qui ne trouvent aucun débouché politique au sein du système institutionnel italien.

On peut également illustrer l'idée d'un lien de proportionnalité inverse entre violence et conflit en revenant à l'expérience contemporaine, en France, des violences urbaines. Celles-ci non 
seulement témoignent du déclin du mouvement ouvrier, je l'ai dit, mais aussi procèdent des difficultés et des lenteurs avec lesquelles se mettent en place de nouvelles conflictualités. Lorsque depuis Marseille, mais aussi depuis les banlieues lyonnaises, est organisée la Marche pour l'égalité et contre le racisme, en 1983, l'action, même si elle comporte une dimension, très minoritaire, de forte tendance à la radicalisation, est avant tout une pression non violente, comparable dans son esprit aux luttes pour les droits civiques dans les Etats-Unis des années soixante. Elle exclut la violence, elle en est l'opposé. Puis son souffle retombe, et bien des émeutes, bien des conduites de rage, notamment dans la banlieue de Lyon, ont à voir avec le sentiment des jeunes qui s'y livrent de n'avoir plus de débouché politique pour une action non-violente. Dans une ville comme Vaulx-en-Velin, la violence urbaine de l'émeute, des rodéos et de la Galère, pour reprendre le titre du livre de François Dubet (Fayard, 1987), a précédé la Marche de 1983, et elle lui succède, marque de grandes carences dans la conflictualisation des demandes des jeunes des quartiers populaires. De même d'ailleurs, la grande émeute de 1990, dans cette même ville, a été suivie par l'apparition ou le renforcement d'associations qui, telles Agora, font clairement le choix de transformer la colère et la rage des jeunes en conflit social et politique, ce qui alimente des relations parfois très tendues avec l'équipe municipale, mais n'a rien à voir avec l'émeute ou les agressions contre des personnes ou des biens. Que l'action conflictuelle succède à la violence oblige, et j'y reviendrai, à envisager une autre piste, qui n'est pas nécessairement contradictoire : celle qui fait de la violence un élément fondateur du conflit, son point de départ, la condition nécessaire pour que se constituent des acteurs, pour que s'exprime une subjectivité jusque-là refoulée, peu explicite aux yeux des protagonistes de la violence eux-mêmes, ou trop inquiète ou malheureuse pour avoir tenté jusque-là de se manifester.

Peut-être même peut-on aller plus loin, en partant du constat d'un phénomène constamment signalé par les observateurs de terrain: l'islam est dans l'ensemble un rempart et non un facteur aggravant de la violence urbaine. Ce constat n'est véritablement démenti que pour la fraction la plus radicalisée, elle-même très limitée, de 
l'islamisme, dont Khaled Kelkal (précisément de Vaulx-en-Velin) fut l'expression la plus connue. En dehors de cette réserve, l'islam, comme mode de socialisation ou de resocialisation, y compris sur un mode plus ou moins revendicatif ou contestataire, propose à ses croyants des normes de comportement qui confinent à l'exemplarité, et un mode d'engagement dans la vie de la Cité qui inclut la conflictualité, la pression négociatrice, mais qui écarte la violence. Là encore, l'existence d'une action structurée idéologiquement (ou religieusement) et aussi pratiquement, et apportant aux croyants les conditions favorables à l'expression collective de leur subjectivité personnelle apparait comme le contraire de la violence.

\section{L'impossible conflictualisation et la violence comme rupture}

Dans l'abondante littérature consacrée à la violence collective, deux penseurs sont souvent associés, qui l'un et l'autre s'intéressent à la subjectivité des acteurs, et pensent la violence en relation au conflit: Georges Sorel et Frantz Fanon. Ce qui vient d'être dit permet en fait de mieux comprendre ce qui les sépare. Pour Georges Sorel, qu'Hannah Arendt (1972) accusait d'amalgamer le marxisme et la philosophie de la vie de Bergson et à qui Jean-Paul Sartre (1961) reprochait des «bavardages fascistes», la violence ouvrière est fondatrice de l'acteur contestataire, à qui elle permet de ne pas s'avachir ni sombrer dans le trade-unionisme. Fondatrice de l'acteur, elle possède selon lui deux vertus. D'une part, la violence ouvrière irait de pair avec l'amélioration de l'action, à qui elle apporterait une «efficacité extraordinaire»; et d'autre part, elle obligerait la bourgeoisie à assumer sa vocation d'acteur dominant en rendant au capitalisme ses «qualités belliqueuses». Laissons de côté la contradiction qu'il y a chez Sorel à évoquer d'un côté la vitalité de la bourgeoisie, retrouvée par la violence, et d'un autre côté sa disparition, qui «n'est plus qu'une affaire de temps». L'intéressant est surtout que Sorel propose une théorie du sujet collectif qui repose sur une forte valorisation de la violence non seulement du point de vue de l'acteur contestataire, non seulement de celui de l'acteur dominant, mais en plus de celui du rapport qui tout à la fois les lie et 
les oppose. Sorel va en effet très loin, en associant cette conflictualité fondée sur la violence à des propositions qui vaudraient pour la civilisation - «la violence (...) apparaît ainsi comme une chose très belle et très héroïque, elle est au service des intérêts primordiaux de la civilisation (...) elle peut sauver le monde de la barbarie» (Sorel, 1981.. p. 110). ${ }^{6}$

Mais appliquée au mouvement social et au conflit, cette théorisation est inacceptable. En France, elle s'est effondrée historiquement, dès l'époque où Sorel écrivait, avec l'éloignement du syndicalisme révolutionnaire de la violence, même sociale, puis avec l'échec en 1908 de ce même syndicalisme qui se caractérisait par une grande radicalité, et sa mutation définitive lors de la guerre de 1914-1918. ${ }^{7}$

Plus généralement, plus le mouvement ouvrier est puissant et efficace comme mouvement social, plus il construit une action organisée et négociatrice, quitte à mener des luttes dures et longues, et plus il ferme l'espace à la violence, qui est toujours dans son histoire la marque de sa crise, de sa faiblesse, voire de sa déstructuration ou de son déclin. L'approche de Sorel apporte une idéologie au mouvement social émergent de son temps, mais il est dans l'erreur s'il s'agit de penser le conflit en tant que relation structurée.

Par contre, là où la violence constitue l'acteur dominé non plus dans une logique de construction d'un rapport conflictuel avec l'acteur dominant, mais dans une logique de rupture, lorsqu'il s'agit de penser non pas le conflit, mais la séparation, lorsque l'image de l'adversaire cède la place à celle de l'ennemi, tout change. C'est ici la pensée du dernier Frantz Fanon, celui des Damnés de la terre (publié l'année de sa mort, en 1961), qu'il faut prendre en considération. Non pas dans certaines de ses outrances ou de ses analyses parfois rhétoriques, par exemple à propos du lumpenproletariat qu'il décrit comme l'avant-garde révolutionnaire dans les villes. Mais lorsqu'il explique que dans l'univers manichéiste de la colonisation, le colonisé doit se constituer de non-homme en homme et que la violence décolonisatrice crée l'acteur, c'est-à-dire l'être humain comme sujet, thème repris et formulé de façon radicale 
par Sartre - la chose colonisée devient homme dans le processus même par lequel elle se libère (p. 3).

Là où le conflit est impossible, là où il s'agit d'opérer la dissociation, la sortie d'un espace politique ou social commun, de rompre, la violence est fondatrice et, dans bien des situations, elle constitue la possibilité la plus directe de l'émancipation qui s'ouvre à l'acteur, comme le montre l'expérience de bien des mouvements de décolonisation ou de libération nationale, même s'il est arrivé aussi dans l'histoire, mais rarement, que se constituent des nations indépendantes et des Etats souverains sans que la violence soit le principal opérateur du changement.

Disons-le nettement : la mise en relation des pensées de Sorel, qui associe à tort rapport social structurel et violence, et de Fanon, qui lie la fin du rapport colonial à la violence, nous conforte dans l'idée que cette dernière est le contraire du conflit, ou bien parce que plus celui-ci est puissamment constitué, et moins elle est présente, ou bien parce qu'elle vient signifier le non-conflit, la rupture, la fin de la relation.

\section{Violence et travail du sens}

Dire de la violence qu'elle est le contraire du conflit, qu'elle trouve un espace d'autant plus large que le rapport conflictuel est lui-même faible ou introuvable tout comme d'ailleurs dire, en adoptant un autre point de vue, qu' elle est le fruit de la crise - sociale, politique, économique, etc.- pourrait laisser supposer une relation de simple proportionnalité inverse, ou suggérer l'image d'un mécanisme élémentaire, voire d'une sorte de jeu à somme nulle. C'est ce que peuvent donner à penser les théories les plus classiques de la sociologie et de la science politique, qu'il s'agisse des approches en termes de frustration, ou de celles en termes de «mobilisation des ressources». ${ }^{8}$ Mais s'en tenir à ce type d'approche revient à passer à côté de l'essentiel et à être aveugle à ce qui caractérise toute violence : le fait que tout à la fois, elle met en œuvre du sens mais que ce sens, 
inévitablement, se perd en elle, s'y dénature, s'y pervertit ou s'y surcharge, ce qui est la marque du travail du sujet à travers la violence où il s'abolit - mais aussi, on l'a vu, se fonde parfois aussi.

Disons-le en termes encore élémentaires : dans toute expérience concrète où la violence intervient, il $\mathrm{y}$ a, plus ou moins spectaculaire, une part d'excès ou de défaut par rapport à ce pourrait être cette expérience si la violence en était absente.

Cette part peut transparaître dans l'idéologie des acteurs, et indiquer par exemple qu'ils parlent en termes artificiels d'une réalité sociale ou culturelle, ou bien encore qu'ils essaient d'intégrer sur un mode mythique, fusionnel, des significations contradictoires ou incompatibles. Ainsi, le terrorisme d'extrême gauche a-t-il été d'autant plus meurtrier, je l'ai dit, qu'il parlait, dans les années soixante-dix et quatre-vingt, au nom d'une classe ouvrière devenant fictive. Différemment, la violence politique d'un mouvement comme ETA, au Pays basque espagnol, a tourné au terrorisme parfois échevelé à partir du moment où il s'efforçait de mettre en forme, de manière de plus en plus irréaliste, la synthèse mythique de ses trois composantes initiales : ETA est devenue d'autant plus terroriste qu'elle parlait au nom, premièrement, d'une identité nationale basque menacée, alors qu'elle était en fait très largement reconnue à partir de la transition démocratique en Espagne, deuxièmement, d'un projet de libération politique ne pouvant plus, dans ce contexte, associer de manière réaliste dans un même combat perspectives démocratiques et pulsions révolutionnaires, et troisièmement, d'un mouvement ouvrier en fait décomposé et très éloigné de se reconnaître dans la pratique des bombes et des enlèvements. La perte de sens, ici apparaît donc comme le produit d'une subjectivité se coupant du réel, d'un rapport concret à l'expérience vécue de ceux auxquels l'acteur de la violence se réfère. Le mythe ou l'idéologie sont le fruit, lui-même instable, de cette déréalisation plus ou moins avancée, qui passe aussi, pour ceux qui en sont les protagonistes, par des processus concrets de séparation et de mise à distance du réel, par exemple à travers l'expérience de la clandestinité. Il serait d'ailleurs intéressant d'aller plus loin dans l'analyse : le mythe par exemple n'est pas lui-même synonyme de 
violence, c'est, du moins dans le cas basque que je viens d'évoquer, lorsqu'il se défait dans la réalité, qu'il devient intenable, que la violence se déploie, vraisemblablement pour le maintenir.

L'excès et le défaut de sens apparaissent aussi dès que l'on considère la présence de la cruauté dans la violence. La cruauté ne s'explique pas, ou que très partiellement, par le souci instrumental de faire régner la terreur. Ainsi, les témoins ou les analystes des récentes épurations ethniques en ex-Yougoslavie ou dans l'Afrique des Grands Lacs soulignent-ils constamment l'association de la cruauté et de la violence, alors même que la cruauté n'est en rien nécessaire à la destruction des personnes. Véronique Nahoum Grappe, à propos de l'ex-Yougoslavie, note que «les excès baroques de la cruauté, sa surenchère énigmatique, gratuite, irrationnelle, n'entrent pas dans la rhétorique de la légitimation d'une politique » et, dans le même ouvrage dirigé par Françoise Héritier (1996, p. 289), Claudine Vidal fait un constat comparable à propos de l'Afrique des Grands Lacs. Primo Levi (1989), pour sa part, à partir de son expérience d'Auschwitz, s'est lui aussi interrogé sur la cruauté inutile où il voyait un des «éléments essentiels de l'hitlérisme», fondé sur l'idée qu' «avant de mourir, la victime doit être dégradée afin que le meurtrier sente moins le poids de sa faute». Remarque éclairante: la cruauté, qui a besoin de l'impunité ou du sentiment d'impunité pour se déployer, nous informe certainement sur la part de subjectivité qui s'inverse dans une certaine pratique de la violence, elle est parfois une manière ou un mécanisme paradoxal pour pouvoir se supporter soi-même. C'est en faisant de l'autre un non-sujet, un être déshumanisé puisqu'il peut être avili et détruit cruellement qu'on se maintient soimême du côté de ce qu'on croit être l'humanité du sujet - un sujet devenu de fait un anti-sujet.

Les conduites d'autodestruction liées à la pratique de la violence nous informent également sur le travail du sens et donc sur la présence d'un sujet qui se transforme pour se mettre à l'épreuve dans la violence. Il en est ainsi, en particulier, dans les phénomènes extrêmes de martyrisme, dont Farhad Khosrokhavar (1998, p. 118) a montré, à propos des «Bassidji», qu'ils peuvent témoigner, 
paradoxalement, d'une pléthore de sens: ces jeunes gens qui, dans l'Iran de Khomeini au début des années quatre-vingt, s'engagent dans la guerre contre l'Irak en sachant fort bien qu'ils y trouveront vraisemblablement la mort sont en quête d'une mort «digne», ils entendent répandre la terreur dans les rangs adverses en même temps que s'y réaliser, et obtenir par leur suicide la réalisation des promesses d'un monde meilleur que la Révolution ne tient déjà plus. Leur religiosité mortifère est le lieu unique où s'exerce une créativité, «la mort est synonyme de salut».

Apparemment au plus loin des conduites d'autodestruction, mais par contre hautement compatibles avec la pratique de la cruauté, certaines violences semblent commandées par la seule recherche du plaisir ou de la jouissance - un plaisir ou une jouissance éventuellement partagés par les témoins ou les spectateurs, lorsqu'ils ne sont pas indifférents ou, autre cas de figure, écoeurés. Norbert Elias, pour rendre compte de la violence des puissants au Moyen Age, introduit ainsi le thème de la jouissance; et pour penser la rupture apportée par le sport moderne, il indique que sa nouveauté est de dissocier le plaisir de la violence et de permettre le «controlled decontrolling of emotions» (Elias et Dunning, 1969). ${ }^{9}$ Mais la recherche du plaisir peut passer aussi par la pratique d'une violence débridée, sinon extrême. On le voit dans les formes les plus radicales de hooliganisme, lorsque des supporters, comme ceux superbement décrits par Bill Buford, n'attendent du match de football que l'occasion d'un déplacement où ils se déchaîneront de manière tribale, bestiale, purement jouissive, contre les partisans de l'équipe adverse, ou contre les forces de l'ordre, en dehors du stade, sans intérêt pour l'événement sportif proprement dit, pour son spectacle ou même pour son résultat. Ici, le sens disparaît entièrement au profit du non-sens, il s'abolit dans la jouissance pure, dans la désocialisation complète du sujet, réduit à son animalité (Buford, 1994).

Mythe et idéologie, cruauté, autodestruction, jouissance : ces aspects de la violence, dont la liste pourrait certainement être allongée, obligent à considérer le sens qui se transforme, se perd ou éventuellement se renouvelle dans l'expérience de la violence. Une 
expérience où le sujet n'est pas tant lui-même perdu ou affaibli que renversé, retourné en autre chose, qu'on peut appeler l'anti-sujet pour marquer qu'on a affaire à une sorte de renversement, à la production d'une figure plus ou moins invertie du sujet.

Cette approche fait du protagoniste de la violence un sujet plus ou moins perverti et nous éloigne de deux types de raisonnements opposés mais qui pourtant ont ceci de commun qu'ils se centrent sur la personnalité de l'acteur. D'une part, elle fait de la violence, lorsqu'elle s'exprime, le point d'aboutissement d'un processus, et elle la définit par l'excès ou le défaut, la surcharge ou le manque de sens ; notre approche se refuse, par conséquent, à naturaliser la violence pour la réduire à l'expression de pulsions, d'affects, elle se refuse à y retrouver, par exemple, une agressivité primordiale n'attendant que le relâchement des contrôles politiques ou moraux pour se libérer. La violence, même si elle revêt l'allure de la pire barbarie, n'est pas le fruit de la désocialisation et de l' affranchissement des contraintes propres à toute vie collective. Elle est l'aboutissement de processus où la part du sens, et donc du social, demeure présente, même si c'est en creux ou de manière particulièrement déformée. Elle n'est pas le retour à l'état de nature, sauf à considérer que la nature est un état particulier de la condition humaine.

Et d'autre part, la violence, dans la perspective qui vient d'être proposée, n'est pas réductible à la traduction, via une structure de personnalité, d'une culture qui lui serait particulièrement propice. Il est vrai qu'une importante tradition de recherches, ne serait-ce qu'à propos du nazisme, et allant alors de Theodor Adorno (1960) à David Goldhagen (1997), insiste sur le terreau culturel qui, en Allemagne, a permis à l'antisémitisme de se déployer sur fond d'une épaisseur historique. Ces pensées sont à l'origine de débats d'une portée considérable, elles nous indiquent qu'une personnalité plus encline que d'autres à la violence peut se construire dans certaines cultures qui lui offrent des conditions favorables. On est avec elles au plus loin de toute naturalisation de la violence, qui devient plutôt un attribut culturel de certaines sociétés ou de certains groupes. Mais la faiblesse, ou les limites de ce type d' approches est qu'elles sous-estiment, voire 
ignorent les processus sociaux et politiques à partir desquels la violence émerge, se développe ou régresse. Ces approches suggèrent un déterminisme culturel où l'on risque de perdre de vue le sens que la violence met en forme (quitte, on l'a dit, à le pervertir). Elles expliquent des événements qui ont lieu à un instant donné par une structure de personnalité ou une culture politique qui leur préexistent avec un décalage dans le temps qui peut être éventuellement considérable, elles sautent au-dessus des médiations et des transformations qui font que la violence n'est jamais inéluctable. Le passage à la violence peut reposer sur des éléments qui renvoient à une personnalité ou à la culture que cette personnalité reproduit ou exprime ; mais, s'il s'agit de la personne, du sujet de la violence, les facteurs les plus importants, très différemment, procèdent du travail de l'acteur sur lui-même, de la production d'une idéologie, d'interprétations changeantes de sa situation, ils relèvent de transformations et non d'une simple reproduction.

\section{La violence fondatrice du sujet}

Déjà en évoquant Frantz Fanon, j'ai pourtant d'une certaine façon mis en avant une hypothèse qui inverse ce qui vient d'être dit : la violence, au lieu d'être le contraire ou l'inversion de la formation ou de l'expression du sujet n'est-elle pas ce qui en autorise l'émergence, l'épanouissement ou la sauvegarde ? La thèse se rencontre couramment dans diverses pensées de l' anthropologie, pour lesquelles la violence serait une condition de la vie collective, incluse notamment dans le sacrifice ou dans les phénomènes de boucémissaire. On la trouve aussi bien chez Sigmund Freud que, plus récemment, chez Walter Burkert ou René Girard. Et à la perspective anthropologique, on peut ici, au moins pour certaines expériences, ajouter une considération plus historique : il est vrai, en effet, que les Etats et les Nations, la plupart du temps, ne se créent que dans le sang et la guerre.

Mais la violence fondatrice n'est pas seulement celle de la collectivité, et donc du sujet collectif, qu'il s'agisse par exemple de 
la tribu, du groupe, de la nation. Son hypothèse mérite d'être aussi évoquée à propos du sujet personnel. Il est vrai que dans certains cas, l'acteur ne semble pouvoir se constituer qu'en s'arrachant à un quotidien fait d'aliénation et de passivité. Que par exemple l'engagement dans une émeute, ou l'entrée dans la délinquance peuvent sembler être des moments décisifs à partir desquels d'autres engagements, et dégagements s'opèreront, violents, ou non violents, amorçant les uns la spirale d'une violence de plus en plus radicale, les autres, au contraire, la participation à des conflits en passe de se structurer. Mais j'ai aussi, lors de mes travaux sur le terrorisme, pu constater que l'idée stéréotypée d'un basculement dans l'engrenage de la violence à partir d'un moment fondateur lui-même violent était couramment démentie par mes interlocuteurs, anciens terroristes qui me décrivaient des processus bien éloignés de correspondre à cette image. Soyons donc ici, simplement, d'une grande prudence: il est possible que la violence soit fondatrice du sujet dans certaines expériences, mais il n'en demeure pas moins qu'elle est souvent, et très vite, son contraire ou sa négation.

\section{Le travail de la violence sur elle-même}

Nous avons déjà dit que pour penser la violence, il convient de penser la place du sujet aboli, interdit, introuvable, la perte de sens, ou l'excès. Cette réponse doit être complétée par l'examen des processus qui s'observent une fois la violence initiée. Que se passe-til, en effet, entre le point de départ d'une expérience de violence, et son aboutissement, quel qu'il soit ? D'innombrables logiques sont ici susceptibles d'opérer, ce qui donne l'image d'une grande variabilité possible de la violence elle-même.

La violence en effet n'est jamais stable bien longtemps, ou stabilisable, contrôlée par son protagoniste, fixée par lui à un seuil ou un autre où serait réglée son intensité. Elle est en elle-même changement, au point qu'on a pu parfois parler pour elle d'engrenage ou de machine infernale. Elle circule du «chaud» au «froid», de l'expressivité sans retenue à l'instrumentalité la mieux organisée - 
mais elle ne s'y installe jamais que provisoirement. Elle passe aussi d'un niveau à un autre, du social au politique, du politique au social, de l'infrapolitique de la délinquance au métapolitique de la religion. Le même acteur peut être un jour terroriste, le lendemain criminel très classique, s'emballer dans des dérives de plus en plus meurtrières et illimitées, ou retourner à une délinquance de faible envergure. Dans les innombrables processus qui font de la violence un phénomène multiforme, celle-ci se décharge et se surcharge aisément de significations qui la transforment constamment. C'est pourquoi son protagoniste peut passer de l'autonomie, c'est-à-dire d'une certaine capacité à fixer lui-même les orientations et les modalités de son action, à l'hétéronomie, qui signifie qu'il devient le vecteur d'un sens qui ne lui appartient pas - à la limite, il devient sicaire, tueur à gage, mercenaire d'une cause qui n'est pas la sienne, ou en tout cas pas celle pour laquelle il a opéré le passage à la violence. Ce qui est le contraire de l'hétéronomie des acteurs sociaux lorsqu'ils sont faibles. Avec la violence, l'acteur hétéronome adopte le sens proposé par d'autres à qui il s' offre comme ressource, tandis que l' acteur social faible demande à d'autres de mettre leurs ressources, par exemple politiques, au service du sens dont il est porteur, mais qu'il ne peut transcrire lui-même dans un rapport conflictuel.

La violence est toujours susceptible de s'éloigner considérablement de son sens premier, et son protagoniste de passer à des conduites où ce sens s'est perdu, tandis qu'un autre, éventuellement, est apparu. Ainsi, pour donner un exemple de cette remarque, le point de départ de Khaled Kelkal, ${ }^{10}$ dont j' ai déjà parlé, est la conviction qu'a ce jeune immigré de subir une discrimination sociale et raciale lui interdisant de se construire personnellement. Le point d'arrivée est une violence extrême, dans laquelle l'islamisme vient apporter un sens pléthorique, mais au plus loin du désir, délaissé ou perdu, de se constituer en sujet personnel dans la société française. Entre les deux, il y eut, sans qu'on ait nécessairement une connaissance précise de sa trajectoire, des épisodes de délinquance, classique, mais aussi la découverte, ou la redécouverte d'un islam l'encourageant à être exemplaire, et à prôner l'exemplarité, notamment à l'égard de ses proches. Mais comprendre le point de départ ne permet pas de 
comprendre le point d'arrivée, ni même la trajectoire. On ne passe pas sans toutes sortes de ruptures et d'inflexions du sens initial de la violence, lourd chez Kelkal d'une subjectivité ancrée dans des rapports sociaux et une expérience vécue qui est celle des jeunes issus de l'immigration dans les banlieues lyonnaises, aux significations qui sont les siennes lorsqu'il s'agit de promouvoir un islamisme devenu radical.

Ce n'est pas parce que le sujet se perd, s'avère introuvable ou impossible au fil de l'expérience d'un acteur, que la recherche doit abandonner l'outil analytique que constitue la notion de sujet. Au contraire, la subjectivité n'est pas une sorte de quantité qui diminuerait au fil des étapes dans la trajectoire de tel ou tel acteur, elle est plutôt ce qui se transforme, se pervertit, s'inverse surtout, dans les cas les plus extrêmes, et que le chercheur doit retrouver dans ses expressions éclatées, appauvries, distordues.

\section{Le point de vue des victimes}

La réflexion sur la violence a longtemps été relativement indifférente aux victimes concrètes. Elle s'est beaucoup plus préoccupée de ses implications générales pour la société dans son ensemble, pour l'ordre social ou moral, pour le tort qu'elle cause aux institutions, étatiques ou autres, familiale notamment.

\section{Une première famille de problèmes}

Le passage à une certaine sensibilité à l'égard des victimes est une caractéristique de la modernité plutôt récente, et qui est un processus en cours, loin d'être achevé. L'action humanitaire en situation de guerre, par exemple, n'est véritablement née qu'avec Henri Dunant et l'invention de la Croix Rouge, dans la seconde moitié du XIXe siècle. La sensibilité à l'égard des victimes du crime ne s'est aiguisée qu'à la même époque ; il en est ainsi, à l'évidence, pour les violences subies par les enfants et les femmes. C'est alors, 
constate Georges Vigarello (1998, p. 186), que le regard sur l'enfant ou la femme victime commence à se transformer, qu'on découvre la violence morale, et qu'on commence à admettre que des pressions et des menaces permettent «d'étendre le territoire de la violence en visant une brutalité non directement physique». Ce mouvement s'est accéléré depuis peu, sous l'effet des fortes mobilisations des mouvements de femmes, ou sous celui de l'opinion publique révulsée par des épisodes comme celui qui, en Belgique, a mis en évidence, tout à la fois, l'ignominie meurtrière de Dutroux et ses complices, et la décomposition des appareils de justice et de répression. Plus largement, la prise en considération du sujet souffrant ou atteint par la violence doit beaucoup à la formation d'acteurs collectifs qui, dans le cadre d'associations notamment, se mobilisent pour obtenir une reconnaissance qui lui est déniée: ainsi, on s'occupe en France beaucoup mieux des victimes du terrorisme, y compris au moment même où le phénomène se manifeste, depuis que Madame Rudetzki, en créant l'association SOS-Attentats, a su mobiliser l'attention des pouvoirs publics et des médias.

Les victimes, on l'admet de plus en plus aujourd'hui, ne sont pas seulement des blessés ou des tués dont on fait la comptabilité de manière administrative, elles sont des sujets plus ou moins atteints dans leur intégrité physique ou morale, privées partiellement ou entièrement, du fait de la violence, d'une capacité à construire leur existence. Le problème n'est pas le même s'il s'agit de victimes d'actes aux dimensions politiques, terrorisme par exemple, ou de crimes, de délinquance, voire d'incivilités : la responsabilité des pouvoirs publics n'est pas la même, et l'expérience vécue diffère nécessairement. Mais dans tous les cas, le point de vue des victimes n'est reconnu que parce que ce qui leur est arrivé est considéré comme un problème concernant la collectivité, et appelant une réponse, un traitement de sa part. C'est pourquoi il est si important que des violences jusque-là méconnues car considérées comme relevant de la seule sphère de la vie privée soient reconnues dans l'espace public : la violence sur les femmes ou les enfants n'est devenue dans bien des pays un problème réel que le jour où elle a été en quelque sorte extraite de l'espace privé. Ce qui conduit à situer aussi les problèmes de la 
violence, et de nos perceptions du phénomène, dans le contexte de la séparation du privé et du public, et des transformations de cette séparation dans les sociétés comme la nôtre. Ce qui conduit également à tracer un parallèle avec le débat contemporain sur le droit d'ingérence. Les changements intellectuels et politiques récents, qui font qu'il est possible aujourd'hui de considérer que si des crimes contre l'humanité sont commis dans un pays, la souveraineté de son Etat passe après le droit d'ingérence de forces internationales, ne procèdent-ils pas d'une logique comparable à celle qui veut que la justice et la police sont concernées par des violences jusque-là ignorées au nom du respect de la vie privée ou de la famille.

\section{Les victimes de crimes contre l'humanité}

Ce qui vaut pour les victimes de la criminalité ou de la délinquance classique vaut aussi pour celles de violences collectives relevant de crime contre l'humanité, et qui, depuis la Seconde guerre mondiale, se dressent, de plus en plus nombreuses, pour réclamer reconnaissance et réparation. Ce phénomène a pris une telle importance qu'on a pu parler de «concurrence des victimes», selon le titre d'un ouvrage récent (Chaumont, 1997), pour rendre compte des dynamiques qui animent divers milieux de mémoire, Juifs, Arméniens, Noirs, etc., au point parfois de les opposer entre elles de manière virulente.

Les victimes, ici, ne sont pas seulement atteintes dans leur être individuel, comme personnes ou sujets personnels. Elles ont aussi été l'objet, elles ou leurs ascendants, de crimes, massacres ou génocides qui ont détruit leurs repères historiques, leur culture, leur mode de vie, d'où par exemple l'emploi d'un terme comme celui d' ethnocide. Et ce qu'elles ont à demander, qu'il s'agisse de reconnaître le drame qu'elles ont vécu ou d'obtenir des compensations ou des réparations, éventuellement tangibles, financières par exemple, ne va pas de soi. Les demandeurs en effet peuvent être les descendants des victimes, et parler en leur nom, quant à ceux à qui s'adressent les demandes, ils peuvent aussi être les héritiers ou descendants des criminels. Que 
signifie alors, par exemple, de se préparer à accorder un pardon au nom de personnes disparues, et à des personnes ou des groupes qui n'ont pas eux-mêmes commis les crimes qu'il s'agit de pardonner? La subjectivité des uns et des autres, ici, est celle de personnes concernées, impliquées, et pourtant qui n'ont, du moins pour certaines d'entre elles ou dans certaines expériences, qu'un rapport historique ou mémoriel avec le drame en question.

\section{Sortir de la violence}

Il est possible de tirer quelques leçons, encore rapides, des analyses qui précèdent, et qui toutes privilégient le point de vue du sujet, pour réfléchir aux prolongements normatifs d'une telle réflexion, et donc à des suggestions relatives à la sortie de la violence, ou à sa régression. Il est possible, autrement dit, de réfléchir à ce qu'on pourrait appeler, à la suite de Robert Fraisse (1997), une politique du sujet appliquée à la violence.

En premier lieu, nous avons insisté sur le fait que la violence se caractérise par la perte de sens. S'il s'agit de recommander des politiques d'intégration, susceptibles d'inclure des exclus ou de liquider les frustrations relatives des plus radicaux, alors, il faut insister sur la nécessité de politiques de reconnaissance, dans lesquelles le problème est d'articuler des efforts pour satisfaire à des demandes ou des attentes d'ordre économique et social, et d'autres permettant de cesser de disqualifier par le mépris et la discrimination sociale et raciste des individus et des groupes. Une politique du sujet, ici, passe par des mesures d'équité sociale et de reconnaissance culturelle, elle peut appeler des efforts de médiation et de restauration de la communication partout où elle se défait et se rompt au profit de logiques de rupture.

Nous avons insisté sur la relation entre violence et conflit : si notre raisonnement est fondé, l'idée d'une politique du sujet, ici, implique que tous les efforts soient déployés pour transformer la violence en conflit. Cela revient à encourager les acteurs en place à 
reconnaître et accepter des interlocuteurs avec qui il vaut mieux échanger: par exemple, pour l'équipe municipale d'une banlieue populaire, d'accepter de débattre et de négocier avec des associations, même très «ethniques» ou religieuses, ou d'encourager des projets portés par des jeunes, mêmes très radicaux, en échangeant avec eux, en favorisant tout ce qui structure et organise leur action sur la durée, même si c'est sur un mode très revendicatif ou hautement conflictuel.

Enfin, nous avons introduit le point de vue des victimes, et il est temps maintenant de l'intégrer dans un raisonnement d'ensemble. La réflexion sur la violence, dans certains cas tout au moins, devrait s'inscrire nettement dans un triangle dont les trois sommets sont constitués par le protagoniste de la violence, la victime, et la collectivité concernée, qu'il s'agisse de la communauté internationale, par exemple en matière de génocide, ou de la nation et de l'Etat, par exemple en matière de délinquance et de crime. Dès lors, la réflexion sur la sortie de la violence, ou sur sa régression devrait, elle aussi, s'inscrire dans un tel triangle. Pour indiquer qu'il s'agit là dune réelle possibilité, on en donnera deux illustrations, l'une renvoyant à la délinquance des mineurs, l'autre à des expériences historiques à implications internationales.

La délinquance des mineurs, lorsqu'elle est traitée selon les modalités de la répression la plus classique, se prolonge couramment par des formes redoublées de violence et de criminalité nées notamment de la détention: les réponses ou les sanctions sont largement criminogènes elles-mêmes. Par ailleurs, les formes les plus classiques de la répression se heurtent à deux problèmes. D'une part, en tant que telles, elles ne se préoccupent en aucune façon des victimes, et d'autre part, elles sont relativement peu soucieuses de la subjectivité malheureuse ou déniée de ceux qui sont devenus délinquants. D’une certaine façon, tout se passe le plus souvent dans la dissociation des trois pôles du triangle évoqué plus haut: la communauté veille à punir le délinquant, et à faire ainsi entendre le discours de l'ordre et de la loi ; la victime est laissée de son côté, quitte à ce que des dommages et intérêts lui soient versés; et le côté «sujet» du délinquant est abandonné aux travailleurs sociaux, ou assimilables, qui n'ont plus 
qu'à opposer leur logique de prévention ou de protection des jeunes à celle de la répression. Or il est possible de chercher à intégrer davantage ces trois dimensions, comme en témoignent les pratiques dites de «réparation» mises en oeuvre dans certains pays, au Canada notamment et, depuis quelques années en France. Il s'agit ici de proposer à la victime d'accepter que le délinquant la dédommage et répare, en commençant par faire connaissance avec elle, et donc par découvrir la personne qu'il a maltraitée ou volée. Ce qui premièrement tient compte de la subjectivité blessée de la victime, deuxièmement implique une sanction et donc la mise en avant de la loi et de la nécessité de respecter ses normes. Ce qui enfin, troisièmement, responsabilise le jeune délinquant, lui permet de retrouver ou de trouver une estime de soi et de fonctionner en sujet responsable ayant reconnu l'autre, sa victime, comme sujet.

Deuxième exemple, celui des négociations permettant de résoudre d'importantes situations proches de la guerre ou de la guerre civile, comme les conflits armés qui séparent Israéliens et Palestiniens, indépendantistes basques et pouvoir espagnol, catholiques et protestants en Irlande du Nord, etc. Il n'y a évidemment pas de recette ou de technique toute faite pour ce type de problèmes, mais l'expérience suggère que si certaines conditions sont remplies, les chances de succès sont plus grandes. Ces conditions impliquent notamment que la violence armée n'interdise pas aux protagonistes de se parler, évidemment de manière discrète, voire secrète, du moins en phase initiale, et qu'ils soient capables de chacun admettre que l'autre, en face, incarne un sujet collectif, méritant reconnaissance. Il faut lire par exemple le récit que donne Uri Savir (1998) des rencontres dites d'Oslo, pour comprendre à quel point cette reconnaissance mutuelle est vitale, et doit être authentique. Le plus important, dans ce récit, tient certainement à la découverte étonnée que font les acteurs de leur vis-à-vis : ils découvrent des êtres humains, sensibles, et non les barbares ou les cyniques qu'ils pouvaient imaginer, ils se perçoivent comme sujets. Et pour que le processus de sortie soit réellement possible, il faut aussi qu'une instance tiers intervienne, extérieure aux acteurs, mais reconnus ou acceptés par eux comme plus ou moins légitime car incarnant un intérêt supérieur transcendant leur propre opposition: ce peut être un responsable politique identifié 
non pas à l'opposition des acteurs, ou à une des parties, mais à l'idée qu'une solution acceptable peut être trouvée par eux - on peut ici rappeler comment Michel Rocard fut l'artisan des accords réglant pour de nombreuses années au moins le drame de Nouvelle Calédonie (il nous a expliqué, lors d'un entretien privé, que le plus important pour lui fut de réunir les deux protagonistes de l'opposition à l'Hôtel Matignon à Paris, puis de leur dire : "il y a de la nourriture dans le frigo", je vous laisse, je suis évidemment à votre disposition, mais c'est à vous de trouver la solution). Ce peut être des diplomates, ou encore un pouvoir politique étranger, capable d'exercer une pression politique ou économique, ou bien encore des responsables d'une institution associée à l'idée même de paix et de résolution négociée de la violence. Ce qui veut dire que la sortie de la violence implique, là encore, la reconnaissance mutuelle que l'autre est un sujet, et l'intervention d'un principe transcendant l'opposition des acteurs face à face, que ce principe soit moral, éthique, ou politique, voire économique.

Il serait absurde de ne plus penser la violence qu'en référence au sujet, qu'il s'agisse de l'acteur ou de la victime. Les conduites humaines en effet ne se déploient pas dans le vide, ou dans le seul choc des subjectivités, mais au sein de systèmes sociaux, politiques, culturels. Mais au sortir d'une époque où la réflexion en général, et à propos de la violence en particulier, a eu tendance à se désintéresser du sujet, au point chez certains d'en proclamer rien moins que la mort, et alors même que manifestement, partout on assiste à un retour du sujet, il me semble qu'en pensant la violence dans la perspective du sujet, on se donne les moyens de mieux réfléchir aux moyens d'y faire face.

\section{Notas}

1 Pour des exemples de bonne tenue, cf. James B. Rule (1988), ou, sur un objet plus limité, Donald J. Shoemaker (1984).

2 On peut ainsi lire l'ouvrage classique de Ted Robert Gurr (Why Men Rebel?) comme une tentative pour combiner les modes d'approche à partir d'un paradigme principal qui est celui de la frustration relative. On peut aussi montrer que les difficultés de Norbert Elias pour penser le nazisme et les phénomènes de violence liés à la décivilisation l'ont ainsi encouragé 
à associer à son mode de raisonnement principal, tel qu'il l'expose dans ses deux grands livres, La civilisation des mours (1973) et La dynamique de l'Occident (1975), des modes d'approche très différents, qu'il juxtapose sans grande capacité d'intégration, ce qui apparaît nettement à la lecture de The Germans. Cf. mon intervention au colloque "Norbert Elias" des 22 et 23 octobre 1999 à l'Université Paris-VII.

3 Cf. Maryse Vaillant (1999). Sur les troubles consécutifs à la guerre et sur les conditions permettant d'y faire face, cf. Louis Cracq (1999).

4 La spécificité de la France apparaît nettement dans la recherche comparative menée par Paola Rebughini (1998).

5 On me permettra, pour tout ce qui concerne le terrorisme, de renvoyer à mes livres Sociétés et terrorisme (1988), et Face au terrorisme (1995).

6 Les expressions citées précédemment proviennent du même ouvrage.

7 Sur cette période de l'histoire du mouvement ouvrier en France, cf. Alain Touraine, Michel Wieviorka et François Dubet (1965).

8 Sur ces approches, que je laisse ici de côté, cf. mon article sur "L'impasse des interprétations classiques" (Wieviorka, 1999).

9 Sur Norbert Elias, cf. Jonathan Fletcher (1997).

10 Tel que le révèle l'entretien publié par Le Monde le 7 octobre 1995, entretien réalisé en 1992 par le sociologue Dietmar Loch.

Abstract: The article aims to develop a theory of violence that is centered on the notion of the subject. The proposed theory does not wish to discard or oppose other possible perspectives, such as those that focus on the rationality of the actor of his frustrations. The point is rather to situate those perspectives withim a more encompassing perspective that runs parallel to them or even subordinates them to the nation of the subject. Privileging the point of view of the subject means fundamentally to explore two ways that frequently cross each other, but that have to be dissociated for the sake of analysis: the first foregrounds the actors of violence, the second its victims. As violence is often a mark of a impeded, forbidden, impossible or unhappy subject, the starting point is a definition of the notion of the subject, of a subject that has also been a victim of violence.

Key-words: urban violence, sociology of the subject, conflict, construction of meaning, theory of violence. 


\section{Bibliographie}

ADORNO, Theodor et al. The authoritarian personality. New York: Mayer, 1960.

ARENDT, Hannah. Du mensonge à la violence: essais de politique contemporaine. Paris: Calmann-Lévy, 1972.

BUFORD, Bill. Parmi les hooligans. Paris: Bourgois, 1994.

CHAUMONT, Jean-Michel. La concurrence des victimes. Paris: La Découverte, 1997.

CRACQ, Louis. Les traumatismes psychiques de guerre. Paris: Odile Jacob, 1999.

ELIAS, Norbert. The Germans. Cambridge: Polity Press, 1996.

. La civilisation des mours. Paris: Calmann-Lévy, 1973.

. La dynamique de l'Occident. Paris: Calmann-Lévy, 1975.

ELIAS. Norbert; DUNNING, Eric. The quest for excitement in leisure. Leisure and Society, n. 2, p. 50-85, 1969.

FLETCHER, Jonathan. Violence and civilization: an introduction to the work of Norbert Elias. Cambridge: Polity Press, 1997.

FRAISSE, Robert. Pour une politique des sujets singuliers. In: PENSER le sujet: autour d'Alain Touraine. Paris: Fayard, 1997. p. 551-564.

GOLDHAGEN, David. Les bourreaux volontaires de Hitler. Paris: Seuil, 1997.

GURR, Ted Robert. Why men rebel? Princeton: Princeton University Press, 1971.

HERITIER, Françoise (Dir.). De la violence. Paris: Odile Jacob, 1996.

JOAS, Hans. La créativité de l'agir. Paris: Cerf, 1999 [1992].

JULLIARD, Jacques. Clémenceau briseur de grèves. Paris: Julliard, 1965.

KHOSROKHAVAR, Farhad. Le modèle Bassidji. Cultures et conflits, Un nouveau paradigme de la violence?(sous la dir. de M.Wieviorka), n. 28/30, 1998.

. L'islamisme et la mort, le martyre révolutionnaire en Iran. Paris:

L'Harmattan, 1995.

. L’utopie sacrifiée: Sociologie de la révolution iranienne. Paris: FNSP, 1993. 
LEVI, Primo. Les naufragés et les rescapés: quarante ans après Auschwitz. Paris: Gallimard, 1989.

REBUGHINI, Paola. La violence juvénile dans les quartiers populaires: étude comparative des périphéries de Lyon et de Milan. Paris: EHESS, 1998. Thèse (Doctorat de Sociologie).

RULE, James B. Theories of civil violence. Berkeley: University of California Press, 1988.

SARTRE, Jean-Paul. [Prefáce]. In: FANNON, Frantz. Damnés de la terre. Paris: Maspéro, 1961.

SAVIR, Uri. The process. New York: Random House, 1998.

SHOEMAKER, Donald J. Theories of delinquency: an examination of explanations of delinquent behavior. New York: Oxford University Press, 1984.

SOREL, Georges. Réflexions sur la violence. Paris: Slatkine, 1981.

TOURAINE, Alain; WIEVIORKA, Michel; DUBET, François. Le mouvement ouvrier. Paris: Fayard, 1984.

VAILLANT, Maryse. La réparation: de la délinquance à la découverte de la responsabilité. Paris: Gallimard, 1999.

VIGARELLO, Georges. L'invention de la violence morale. Sociétés et représentations, n. 6, p.186, juin 1998.

WIEVIORKA, Michel. Face au terrorisme. Paris: Liana Levi, 1995.

. L'impasse des interprétations classiques. Le Monde des Débats, n. 7, p. 10-13, octobre 1999. . Sociétés et terrorisme. Paris: Fayard, 1988. 